\title{
Comparison of microbiology and visual outcomes of patients undergoing small-gauge and 20-gauge vitrectomy for endophthalmitis
}

This article was published in the following Dove Press journal:

Clinical Ophthalmology

22 January 2016

Number of times this article has been viewed

\author{
David RP Almeida' \\ Eric K Chin ${ }^{2,3}$ \\ Shaival S Shah ${ }^{3}$ \\ Benjamin Bakall ${ }^{3}$ \\ Karen M Gehrs ${ }^{3}$ \\ $\mathrm{H}$ Culver Boldt ${ }^{3}$ \\ Stephen R Russell ${ }^{3}$ \\ James C Folk ${ }^{3}$ \\ Vinit B Mahajan ${ }^{3,4}$ \\ 'VitreoRetinal Surgery PA, \\ Minneapolis, MN, ${ }^{2}$ Retina Consultants \\ of Southern California, Riverside, CA, \\ ${ }^{3}$ Vitreoretinal Service, Department of \\ Ophthalmology and Visual Sciences, \\ ${ }^{4}$ Omics Laboratory, University of \\ lowa, lowa City, IA, USA
}

Correspondence: Vinit B Mahajan Omics Laboratory, University of lowa, 200 Hawkins Drive, lowa City, IA 52242, USA

$\mathrm{Tel}+\mathrm{I} 3193563185$

$\mathrm{Fax}+$ I 3193560363

Email vinit-mahajan@uiowa.edu
Background: The role of pars plana vitrectomy (PPV) for endophthalmitis has evolved over recent decades but the literature is lacking on comparisons between small-gauge and 20-gauge vitrectomy.

Objective: To evaluate evolving etiological and microbiological trends in patients undergoing vitrectomy for endophthalmitis and to compare culture-positive rates and visual outcomes between small-gauge (23- and 25-gauge) and 20-gauge instrumentation during vitrectomy for endophthalmitis.

Methods: Ten-year retrospective comparative case series and prospective laboratory in vitro testing. Tertiary care academic referral center. Patients who underwent PPV for endophthalmitis between 2003 and 2013. Vitreous biopsies were obtained in all cases. The effect of vitrectomy gauge (20-, 23-, and 25-gauge) and vitreous cutting rate (1,500 and 5,000 cuts per minute) on the viability of bacterial culture was evaluated in an in vitro prospective laboratory investigation.

Main outcome measures: Comparison of etiology, microbiology culture-positive rates, and visual outcomes between small-gauge and 20-gauge instrumentation in patients undergoing PPV for infectious endophthalmitis.

Results: A total of 61 cases of vitrectomy for endophthalmitis were identified over a 10-year period; of these, 34 were treated with small-gauge (23- and 25-gauge) vitrectomy and 27 were treated with 20 -gauge vitrectomy. In the small-gauge group, 12 cases (35.3\%) yielded culture-positive results versus 20 cases $(74.1 \%)$ with culture positivity in the 20 -gauge cohort $(P=0.002)$. The most common cause of endophthalmitis was cataract surgery and the most frequently identified organism was coagulase-negative Staphylococci in both groups. There was no significant difference in mean postoperative visual acuities between groups $(P=0.33)$. Etiological trends indicate an increase in endophthalmitis due to intravitreal injection in the small-gauge group $(n=9)$ compared to the 20-gauge group $(n=3)(P=0.001)$. In vitro laboratory testing revealed no significant difference in rates of culture growth for different vitrectomy gauge sizes or vitreous cutting speeds.

Conclusion and relevance: Small-gauge vitrectomy for endophthalmitis yields final visual outcomes comparable to 20 -gauge instrumentation. A significant difference in culture-positive rates was observed between small-gauge and 20-gauge instrumentation for vitrectomy in endophthalmitis; however, laboratory testing indicates this is not related to either vitreous gauge size or cutter speed. Intravitreal injections are emerging as a common etiology of vitrectomy for endophthalmitis.

Keywords: endophthalmitis, vitrectomy, vitreous biopsy

\section{Introduction}

Endophthalmitis is a potentially devastating sight-threatening intraocular inflammatory disorder resulting from infection of the vitreous cavity. The role of pars plana 
vitrectomy (PPV) for endophthalmitis has evolved over recent decades, particularly after the Endophthalmitis Vitrectomy Study (EVS), and tends to be reserved for advanced cases. ${ }^{1,2}$ PPV is believed to help in the treatment of endophthalmitis by removal of infecting organisms and toxins, removal of vitreous membranes that could otherwise lead to retinal detachment, clearing of vitreous opacities, allowing better penetration of injected antibiotics, and collection of adequate vitreous material for subsequent culture.

Since the EVS, recent advancements in microsurgical instrumentation have led to the increased use of smaller gauge, specifically 23- and 25-gauge, instrumentation for PPV. ${ }^{3-5}$ In contrast to 20 -gauge vitrectomy, smaller-gauge surgery utilizes sclerotomies that are created without conjunctival dissection, utilize a cannula, and are typically left unsutured. These small gauge wounds offer the potential advantages of reduced operative time, decreased surgical trauma, reduced postoperative astigmatism, and more rapid postoperative visual recovery. ${ }^{6,7}$

While both 23- and 25-gauge instrumentation are currently widely used for vitreoretinal procedures, ${ }^{8-15}$ the literature is lacking on any comparison between small-gauge and 20-gauge vitrectomy in the setting of endophthalmitis. Additionally, there is some anecdotal concern that small gauge instrumentation may not provide as viable microbiological specimens, owing to smaller vitrector lumen size and increased vitreous cutter speed, when compared to 20-gauge instrumentation. We set out to compare the etiology, microbiology culture positivity rates, and visual outcomes between small-gauge and 20-gauge instrumentation in patients undergoing vitrectomy for endophthalmitis over a 10-year period at the University of Iowa. Additionally, we performed in vitro laboratory testing to investigate the effects of vitrector lumen size and cutter speed on the ability to culture colonies of bacteria.

\section{Methods}

\section{Retrospective comparative case series}

The study protocol was approved by the Institutional Review Board for Human Subjects Research at the University of Iowa and adheres to the tenets set forth in the Declaration of Helsinki. A retrospective comparative case series of patients who underwent PPV for the diagnosis of endophthalmitis between 2003 and 2013 was conducted. Patient cases were divided into small gauge (23- and 25-gauge) and 20-gauge groups. Demographic data, pre- and postoperative visual acuity, culture results, and clinical course were reviewed. Urgent PPV was performed in eyes presenting with light perception vision or worse and clinical signs of endophthalmitis after cataract surgery as per the recommendations of the EVS. Eyes with hand motions vision or better had PPV performed at the discretion of the attending surgeon based on etiology, ocular history, and prognosis. All eyes had a minimum follow-up duration of 1 month. All patients underwent a minimum examination schedule of postoperative day 1, week 1, and month 1. At each postoperative visit, Snellen visual acuity and intraocular pressure in the operative eye were recorded and a complete ophthalmic examination was performed. Statistical analysis was performed using Student $t$-tests of independent proportions. No consent was needed for this retrospective review as approved by the University of Iowa Research Ethics Board.

\section{In vitro laboratory testing}

Staphylococcus epidermidis bacteria were obtained (ATCC 12228; ATCC Manassas, VA, USA) and the concentration of colony forming units (cfu) was calculated by performing a growth curve (replication rate). A suspension of $S$. epidermidis in $5 \mathrm{~mL}$ LB (Luria broth) was incubated at $37^{\circ} \mathrm{C}$ overnight. Following incubation, $500 \mu \mathrm{L}$ of the suspension was added to a vial containing $4.5 \mathrm{~mL} \mathrm{LB}$ medium and maintained at $37^{\circ} \mathrm{C}$. A sample of $100 \mu \mathrm{L}$ was removed once every hour for optical density measurement at $600 \mathrm{~nm}$ using a Nanodrop ND-1000 spectrophotometer (NanoDrop products, Wilmington, DE, USA). A series of serial dilutions were generated by placing $50 \mu \mathrm{L}$ aliquot of the removed suspension in $450 \mu \mathrm{L}$ LB medium. After using standard dilution techniques, a $100 \mu \mathrm{L}$ of each diluted sample was plated on duplicate LB agar plates to provide a suitable density for colony counts (30-300 colonies/plate). A bent sterile glass rod was dipped in the sample and brushed over the plate while the plate was rotated in a circular motion. All plates were incubated for 24 hours at $37^{\circ} \mathrm{C}$ before the colonies were counted. The concentration of viable colonies was calculated and correlated to the optical density measurement. An optical density of 0.16 was found to correspond to a concentration of $3.3 \times 10^{8} \mathrm{cfu} / \mathrm{mL}$, and this optical density was used as a reference to prepare the diluted bacterial suspension of $10^{6} \mathrm{cfu} / \mathrm{mL}$ for the experiment.

The cultured and diluted suspensions were placed in several $2.0 \mathrm{~mL}$ centrifuge tubes, and samples of $1.4 \mathrm{~mL}$ each were collected through three different sizes of vitrectomy hand pieces (20-, 23-, and 25-gauge) connected to a Constellation Vision System (Alcon, Fort Worth, TX, USA). Cutting rates were set at zero (aspiration only), 1,500 cuts per minute (cpm), and 5,000 $\mathrm{cpm}$ (the aspiration rate was set to $200 \mathrm{mmHg}$ for 
all samples). After the sample collection, the vitrectomy tubing was disconnected and flushed multiple times with sterile balanced salt solution (BSS) followed by drying of the interior of the tube with filtered air. An aliquot of the BSS flushed through the tubing was saved as a control sample. A new set of vitrectomy hand pieces were used for the two cutting speeds $(1,500$ and 5,000 cpm) for each vitrector gauge. All the collected aliquots were immediately processed for serial dilution, cultured on LB agar plates, incubated for 24 hours, and viable colonies were counted as described earlier.

\section{Surgical technique}

Small-gauge and 20-gauge vitrectomies were performed either with retrobulbar anesthesia under monitored anesthesia care or under general anesthesia. Periorbital skin and eyes were prepped with $5 \%$ povidone-iodine (Betadine; Purdue Fredrick Co., Norwalk, CT, USA). Beveled sclerotomy wounds were created with a single-step Alcon 23-gauge or 25-gauge trocar/cannula microvitrectomy system. In 20 -gauge cases, a microvitreoretinal blade was used to create sclerotomies following conjunctival dissection. The vitreous cutter was inserted and an undiluted vitreous sample was collected and sent for culture. The vitrector was sometimes used to remove fibrin and pupillary membranes in the anterior chamber. Direct visualization and a noncontact wide field viewing system (BIOM; Oculus Inc., Wetzlar, Germany) were used for visualization. A core vitrectomy was performed in all cases and $0.1 \mathrm{cc}$ of intravitreal vancomycin $(1 \mathrm{mg} / 0.1 \mathrm{~mL})$ and $0.1 \mathrm{cc}$ of ceftazidime $(2.25 \mathrm{mg} / 0.1 \mathrm{~mL})$ were injected at the conclusion of surgery.

\section{Results}

Between 2003 and 2013, a total of 61 cases of infectious endophthalmitis were managed with primary vitrectomy at the University of Iowa. In the 20-gauge group, 27 cases of endophthalmitis were analyzed with a mean follow-up of 26 months (range 1-90 months). In contrast, there were 34 cases evaluated in the small-gauge group consisting of 23 -gauge $(n=12)$ and 25 -gauge $(n=22)$ instrumentation; the mean follow-up was 9.3 months (range 1-36 months). No significant intraoperative complications were reported.

The most common cause of infectious endophthalmitis in both groups was post-cataract extraction ( $\mathrm{n}=9$ in both groups). Other common causes included: bleb-related endophthalmitis, post-intravitreal injection, and endogenous endophthalmitis (Figure 1). There was a significant increase in number of cases of endophthalmitis due to intravitreal injections in the small-gauge group $(n=9)$ compared to the 20 -gauge group $(\mathrm{n}=3)(P=0.001)$. Post-intravitreal injection endophthalmitis consisted of corticosteroid $(\mathrm{n}=1)$ and anti$\operatorname{VEGF}(\mathrm{n}=2)$ in the 20-gauge group compared to corticosteroid $(n=5)$ and anti-VEGF $(n=4)$ in the small-gauge group.

Preoperative visual acuity for all patients ranged from $20 / 80$ to light perception. The final postoperative visual acuity ranged from $20 / 20$ to no light perception (NLP); the best outcomes were encountered in post-cataract cases whereas the worst outcomes occurred in cases of bleb-related endophthalmitis. The final visual outcomes were similar with a significant portion of patients achieving a final visual acuity of 20/40 or better in both the small-gauge $(n=9,26.5 \%)$ and 20-gauge $(\mathrm{n}=8,29.6 \%)$ groups $(P=0.3)($ Table 1$)$.
A

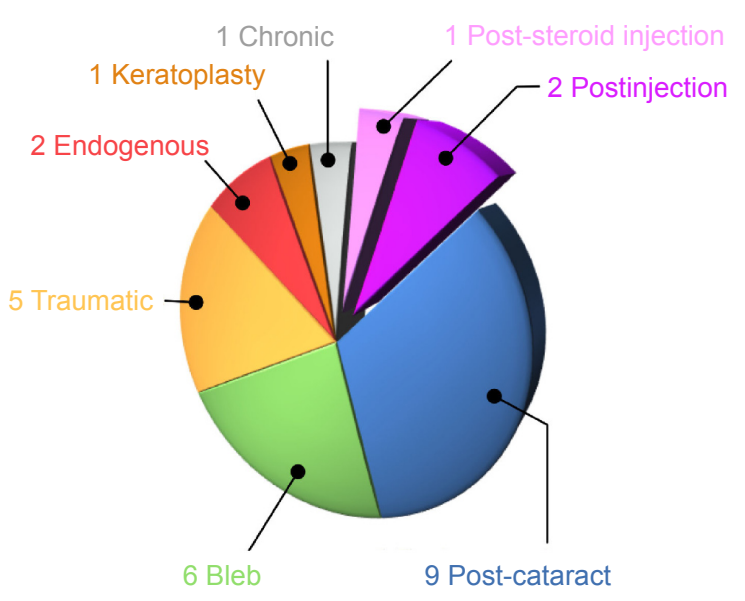

B

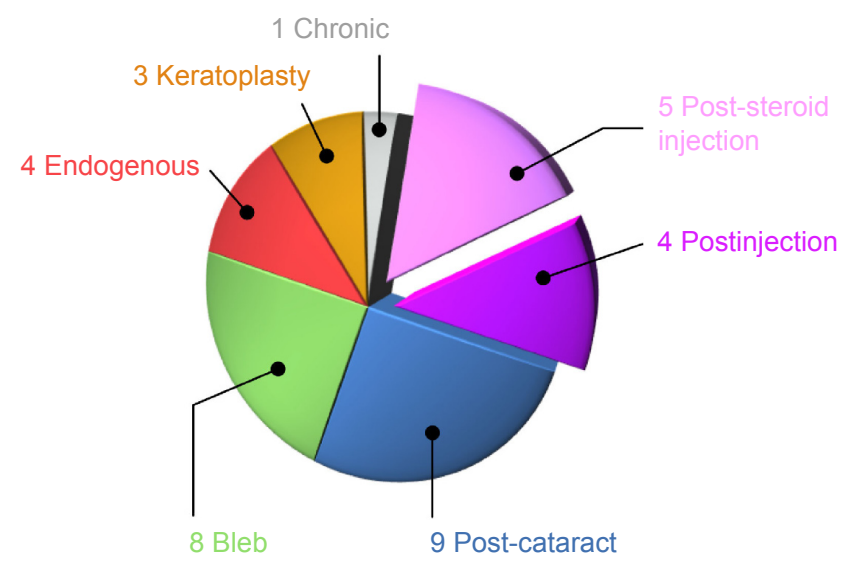

Figure I Causes of infectious endophthalmitis.

Notes: All causes of infectious endophthalmitis for 20-gauge (A) and small-gauge (B) vitrectomy groups are presented. There were an increasing number of cases of postintravitreal injection endophthalmitis in the small-gauge group. Numbers I to 9 represent number of cases. 
Table I Final postoperative visual outcomes

\begin{tabular}{llll}
\hline Final visual acuity & Small-gauge* & 20-gauge* & $P$-value \\
\hline 20/40 or better & 9 of $34(26.5 \%)$ & 8 of $27(29.6 \%)$ & 0.33 \\
20/100 or better & 16 of $34(47.1 \%)$ & 12 of $27(44.4 \%)$ & 0.34 \\
$5 / 200$ or better & 23 of $34(67.6 \%)$ & 13 of $27(48.1 \%)$ & 0.17 \\
\hline
\end{tabular}

Notes: Final stratified visual acuities for small gauge (23- and 25-gauge) and 20gauge vitrectomy for infectious endophthalmitis. *Indicates number of cases out of total number of cases.

Microbiological etiology was similar in both groups with coagulase-negative Staphylococci being the most commonly isolated bacteria (Figure 2). There was a statistically significant difference in culture positivity rates of vitreous biopsies between 20 -gauge ( 20 of $27,74.1 \%$ ) compared with smallgauge $(12$ of $34,35.3 \%)$ instrumentation $(P=0.002)$. The dramatic differences in culture-positive rates between groups raised the hypothesis of possible causes relating to vitreous cutter lumen size and cutting speed; consequently, a prospective in vitro laboratory experiment was designed to determine the effect of gauge size and cutter speed on bacterial culture.

Biopsy samples from normalized counts of $S$. epidermidis cultures were collected using 20-, 23-, and 25-gauge vitrectors set at 1,500 and 5,000 cpm. As expected, there were no identified colonies growing from the BSS control and negative control. Manual aspiration without cutting yielded a significant number of colonies (Figure 3). Cultures yielded viable colonies after 24 hours of growth for all gauge instruments and cutting speeds tested. There were no statistically significant differences in bacterial culture growth for the aspirated sample with a cut rate of zero, 1,500 , or $5,000 \mathrm{cpm}(P=0.39)$. In addition, there were no statistically significant differences in the culture yield for any of the different vitrector gauges tested $(P=0.38)$ (Figure 3 ). The most robust culture growth was seen with
20 -gauge vitrector set at $1,500 \mathrm{cpm}$ and 25 -gauge vitrector at $5,000 \mathrm{cpm}$.

\section{Discussion}

Small-gauge vitrectomy is effective in the treatment of many retinal diseases with advantages over 20 -gauge instrumentation such as: greater ease of insertion into the anterior chamber to remove papillary membranes, less postoperative inflammation, and decreased surgical time. ${ }^{16,17} \mathrm{We}$ find that, in cases of endophthalmitis requiring vitrectomy, final visual outcomes are similar for both small-gauge and 20 -gauge vitrectomy.

When stratifying outcomes based on etiology, we see that in both groups, poor outcomes were associated with cases of bleb-associated endophthalmitis and related to severe organism virulence. The increased incidence of Streptococcus species and Gram negative species in these groups has been previously documented and is associated with poor visual outcomes. ${ }^{18,19}$ In the present study, 14 of 61 (23\%) cases were bleb-associated endophthalmitis and, of these, 14 cases (71\%) had a final visual acuity worse than 5/200. Furthermore, of the 14 total cases of bleb-associated endophthalmitis in our study, six of the 14 were treated with 20 -gauge vitrectomy with four (67\%) having a final visual acuity worse than 5/200. Comparatively, of the eight bled-associated endophthalmitis cases treated with small-gauge vitrectomy, six (75\%) had final visual acuities worse than 5/200. Although the total numbers of cases were small, there was no significant difference between the two groups, which mirrors our visual acuity outcome data presented earlier.

Our data is congruent with increasing trends of intravitreal injections as a major cause of endophthalmitis. ${ }^{20}$ This represents a significant shift in the cause of endophthalmitis

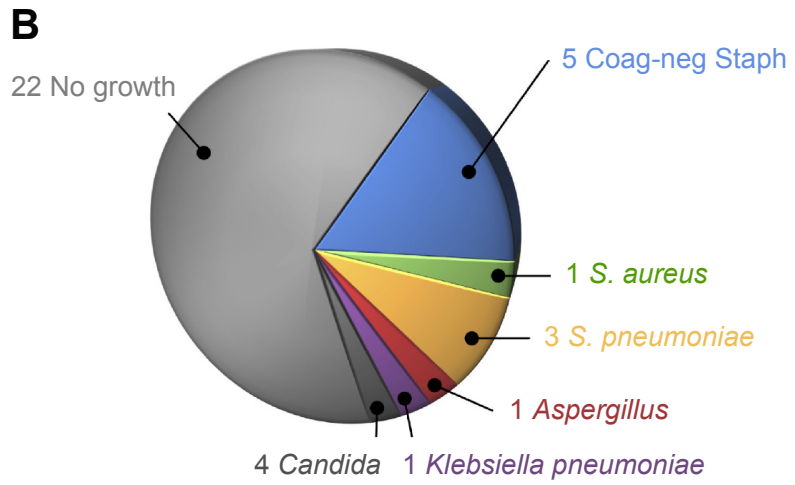

Figure 2 Microbiological etiology of infectious endophthalmitis.

Notes: Coagulase-negative Staphylococcus (Coag-neg Staph) and Streptococcus pneumoniae were the most frequently cultured organisms in both groups. There was a significant difference in culture positivity rates between 20-gauge (A) and small-gauge (B) vitrectomy groups. Numbers I to 9 represent number of cases.

Abbreviations: S. aureus, Staphylococcus aureus; S. pneumoniae, Streptococcus pneumoniae. 


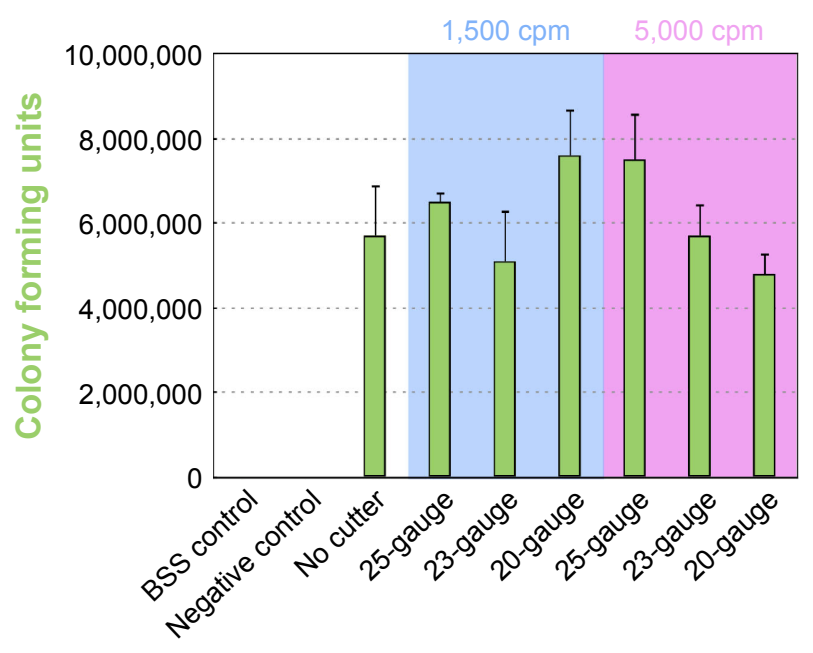

Figure 3 In vitro culture results.

Notes: Staphylococcus epidermidis colony forming units (cfu) were assayed for each vitrectomy lumen size (25-, 23-, and 20-gauge) at both I,500 and 5,000 cuts per minute (cpm) (fixed cut) compared to balanced salt solution (BSS) and negative controls. There was no statistically significant difference in culture growth rates for either vitrector size or vitrector cutting speed. Error bars represent standard deviation.

compared to previous studies ${ }^{19}$ and is related primarily to the increased number of intravitreal injections now performed worldwide for a myriad of retinal conditions. Our small-gauge vitrectomy group has nine cases of endophthalmitis due to intravitreal injection and was similar in prevalence to postcataract surgery $(n=9)$ and bled-associated endophthalmitis $(n=8)$. Based on our data, we feel primary vitrectomy with small-gauge vitrectomy is an adequate approach for severe cases of endophthalmitis after intravitreal injection and provides similar visual outcomes at 20 -gauge vitrectomy.

Although final visual outcomes were similar between small-gauge and 20-gauge vitrectomy cases, there was a marked difference in culture positivity rates between smallgauge (35.3\%) and the 20 -gauge $(74.1 \%)$ groups $(P=0.002)$ from our 10-year data set. The EVS found a $69.3 \%$ culturepositive rate using 20 -gauge vitrectomy, ${ }^{2}$ which compares favorably to our $74.1 \%$; however, we found a much lower culture-positive rate in the small-gauge group. One possibility for this discrepancy is the possibility of an increased number of sterile endophthalmitis cases in the small-gauge group, which have been linked to intravitreal injections. ${ }^{21-23}$ In the small-gauge group, seven of the nine intravitreal injection cases were culture-negative; however, if one were to assume that all these were sterile inflammatory reactions, the overall culture-positive rate would only increase to $44.4 \%$ in the small-gauge group, which would still remain much lower than the 20-gauge cohort.

A further possibility for the lower culture positivity rate in the small-gauge group could be related to a change in laboratory practices that coincided with our study period. After reviewing microbiology standards and practices at the University of Iowa, there was no indication that any significant change had occurred in the protocols for culturing samples from endophthalmitis cases during our 10-year analysis. Previously, it has been shown that microbiology concordance is lacking between anterior chamber and vitreous samples in cases of endophthalmitis, ${ }^{24}$ but this could not be the case in our current study as all samples were derived solely from the vitreous cavity.

It is possible that the decreased culture positivity rate in small-gauge vitrectomy was due to surgeon's learning curve moving away from 20 -gauge instrumentation. In other words, surgeons switching to small-gauge vitrectomy could have difficulties in early cases leading to compromised microbiological yields. Running counter to this is the fact that an undiluted sample was obtained at the beginning of the case, thereby limiting any downstream surgical step from affecting the endophthalmitis microbiological sample.

To further investigate the low culture yield in small-gauge cases, we set out to test the effect of vitrector size (20-, 23-, and 25-gauge) and cutter speed (1,500 and 5,000 cpm) on the ability to culture bacteria with a prospective laboratory in vitro experiment. Using 20 -gauge vitrectomy, previous studies have shown that there is no significant difference in the microbiologic yield between vitreous needle aspiration and a mechanized vitreous cutter with cutting rates of $1,500 \mathrm{cpm} .{ }^{25,26}$ Currently, there are no such studies in the literature comparing older 20-gauge systems with newer vitrectomy systems; in spite of this, there is regular anecdotal discussion that small-gauge vitrectomy units, with higher cut rates of 5,000 cpm, use higher vacuum and create higher shear forces that may decrease the microbiological yield. Our in vitro experiments suggest that neither instrument gauge nor cutting rate significantly affect the ability to culture a bacterial organism. Interestingly, the largest microbiological bacterial yield was found at $1,500 \mathrm{cpm}$ with a 20 -gauge vitrector and 5,000 cpm with a 25 -gauge vitrector showing that both systems adequately allow for excellent culture growth rates.

There are several limitations to our study. First, it is a retrospective case-control study. Second, although our series is moderately large, a prospective trial with larger sample sizes could yield additional or even different results. Third, duration of follow-up was variable. We have tried to mitigate these three limitations by having a relatively large sample size over a 10-year period at one institution to limit further confounders. 
In conclusion, small-gauge vitrectomy yields final visual outcomes comparable to 20-gauge instrumentation in cases of infectious endophthalmitis. Intravitreal injections are emerging as a common etiology of vitrectomy for endophthalmitis, and this is likely to continue to rise in incidence. We found a significant difference in culture-positive rates between small-gauge and 20-gauge instrumentation for vitrectomy in endophthalmitis, which, based on laboratory testing, indicates this is not related to either vitreous gauge size or cutter speed. Possible other causes for the discrepancy in culture-positive rates, such as effect of vitrectomy tubing, should be studied further.

\section{Acknowledgment}

Unrestricted grant from Research to Prevent Blindness.

\section{Disclosure}

The authors report no conflicts of interest in this work.

\section{References}

1. Machemer R, Buettner H, Norton EW, Parel JM. Vitrectomy: a pars plana approach. Trans Am Acad Ophthalmol Otolaryngol. 1971;75(4): 813-820.

2. Results of the Endophthalmitis Vitrectomy Study. A randomized trial of immediate vitrectomy and of intravenous antibiotics for the treatment of postoperative bacterial endophthalmitis. Endophthalmitis Vitrectomy Study Group. Arch Ophthalmol. 1995;113(12):1479-1496.

3. Eckardt C. Transconjunctival sutureless 23-gauge vitrectomy. Retina. 2005;25(2):208-211.

4. Fujii GY, De Juan E Jr, Humayun MS, et al. Initial experience using the transconjunctival sutureless vitrectomy system for vitreoretinal surgery. Ophthalmology. 2002;109(10):1814-1820.

5. Fujii GY, De Juan E Jr, Humayun MS, et al. A new 25-gauge instrument system for transconjunctival sutureless vitrectomy surgery. Ophthalmology. 2002;109(10):1807-1812; discussion 1813.

6. Misra A, Ho-Yen G, Burton RL. 23-gauge sutureless vitrectomy and 20-gauge vitrectomy: a case series comparison. Eye (Lond). 2009;23(5): $1187-1191$.

7. Spirn MJ. Comparison of 25, 23 and 20-gauge vitrectomy. Curr Opin Ophthalmol. 2009;20(3):195-199.

8. Fine HF, Iranmanesh R, Iturralde D, Spaide RF. Outcomes of 77 consecutive cases of 23-gauge transconjunctival vitrectomy surgery for posterior segment disease. Ophthalmology. 2007;114(6):1197-1200.
9. Patelli F, Radice P, Zumbo G, Frisone G, Fasolino G. 25-gauge macular surgery: results and complications. Retina. 2007;27(6):750-754.

10. Kadonosono K, Yamakawa T, Uchio E, Yanagi Y, Tamaki Y, Araie M. Comparison of visual function after epiretinal membrane removal by 20-gauge and 25-gauge vitrectomy. Am J Ophthalmol. 2006;142(3): 513-515.

11. Rizzo S, Belting C, Cresti F, Genovesi-Ebert F. Sutureless 25-gauge vitrectomy for idiopathic macular hole repair. Graefes Arch Clin Exp Ophthalmol. 2007;245(10):1437-1440.

12. Lakhanpal RR, Humayun MS, de Juan E Jr, et al. Outcomes of 140 consecutive cases of 25-gauge transconjunctival surgery for posterior segment disease. Ophthalmology. 2005;112(5):817-824.

13. Altan T, Acar N, Kapran Z, Unver YB, Ozdogan S. Transconjunctival 25 -gauge sutureless vitrectomy and silicone oil injection in diabetic tractional retinal detachment. Retina. 2008;28(9):1201-1206.

14. Gonzales CR, Singh S, Schwartz SD. 25-Gauge vitrectomy for paediatric vitreoretinal conditions. Br J Ophthalmol. 2009;93(6):787-790.

15. Kiss S, Vavvas D. 25-gauge transconjunctival sutureless pars plana vitrectomy for the removal of retained lens fragments and intraocular foreign bodies. Retina. 2008;28(9):1346-1351.

16. Tarantola RM, Graff JM, Somani R, Mahajan VB. Temporal approach for small-gauge pars plana vitrectomy combined with anterior segment surgery. Retina. 2012;32(8):1614-1623.

17. Mahajan VB, Tarantola RM, Graff JM, et al. Sutureless triplanar sclerotomy for 23-gauge vitrectomy. Arch Ophthalmol. 2011;129(5): 585-590.

18. Leng T, Miller D, Flynn HW Jr, Jacobs DJ, Gedde SJ. Delayed-onset bleb-associated endophthalmitis (1996-2008): causative organisms and visual acuity outcomes. Retina. 2011;31(2):344-352.

19. Busbee BG, Recchia FM, Kaiser R, Nagra P, Rosenblatt B, Pearlman RB. Bleb-associated endophthalmitis: clinical characteristics and visual outcomes. Ophthalmology. 2004;111(8):1495-1503.

20. Folk JC, Stone EM. Ranibizumab therapy for neovascular age-related macular degeneration. N Engl J Med. 2010;363(17):1648-1655.

21. Kay CN, Tarantola RM, Gehrs KM, et al. Uveitis following intravitreal bevacizumab: a non-infectious cluster. Ophthalmic Surg Lasers Imaging. 2011;42(4):292-296.

22. Taban M, Singh RP, Chung JY, Lowder CY, Perez VL, Kaiser PK. Sterile endophthalmitis after intravitreal triamcinolone: a possible association with uveitis. Am J Ophthalmol. 2007;144:50-54.

23. Mahajan VB, Folk JC, Boldt HC. A head-tilt test for hypopyon after intravitreal triamcinolone. Retina. 2009;29(4):560-561.

24. Almeida DR, Miller D, Alfonso EC. Anterior chamber and vitreous concordance in endophthalmitis: implications for prophylaxis. Arch Ophthalmol. 2010;128(9):1136-1139.

25. Ratanapojnard T, Roy CR, Gariano RF. Effect of vitrector cutting rate on vitreous biopsy yield. Retina. 2005;25(6):795-797.

26. Han DP, Wisniewski SR, Kelsey SF, Doft BH, Barza M, Pavan PR. Microbiologic yields and complication rates of vitreous needle aspiration versus mechanized vitreous biopsy in the Endophthalmitis Vitrectomy Study. Retina. 1999;19(2):98-102.
Clinical Ophthalmology

\section{Publish your work in this journal}

Clinical Ophthalmology is an international, peer-reviewed journal covering all subspecialties within ophthalmology. Key topics include: Optometry; Visual science; Pharmacology and drug therapy in eye diseases; Basic Sciences; Primary and Secondary eye care; Patient Safety and Quality of Care Improvements. This journal is indexed on Submit your manuscript here: http://www.dovepress.com/clinical-ophthalmology-journal

\section{Dovepress}

PubMed Central and CAS, and is the official journal of The Society of Clinical Ophthalmology (SCO). The manuscript management system is completely online and includes a very quick and fair peer-review system, which is all easy to use. Visit http://www.dovepress.com/ testimonials.php to read real quotes from published authors. 